\title{
A EQUAÇÃO-MESTRA: ATINGINDO O EQUILÍBRIO
}

\author{
Thiago Carita Correra* e André de Carvalho Frank \\ Instituto de Química, Universidade de São Paulo, CP 26077, 05513-970 São Paulo - SP, Brasil
}

Recebido em 6/5/10; aceito em 23/8/10; publicado na web em 7/1/11

\begin{abstract}
THE MASTER EQUATION: REACHING THE EQUILIBRIUM. In the last years, a great interest in nonequilibrium systems has been witnessed. Although the Master Equations are one of the most common methods used to describe these systems, the literature about these equations is not straightforward due to the mathematical framework used in their derivations. The goals of this work are to present the physical concepts behind the Master Equations development and to discuss their basic proprieties via a matrix approach. It is also shown how the Master Equations can be used to model typical nonequilibrium processes like multi-wells chemical reactions and radiation absorption processes.
\end{abstract}

Keywords: Master Equation; chemical kinetics; radiation absorption.

\section{INTRODUÇÃO}

Por mais que os físico-químicos desejem que os sistemas estejam em equilíbrio, a natureza não está em equilíbrio. Toda a vida depende de uma força motriz que, por sua vez, é gerada por sistemas fora do equilíbrio. Por exemplo, tanto o movimento dos mares como o fluxo de energia entre corpos a temperaturas distintas, como o Sol e o nosso planeta, são baseados em processos fora do equilíbrio.

Estes fenômenos fazem com que a necessidade de se estudar processos fora do equilíbrio seja inquestionável. Apesar disso, pouco é discutido a respeito desses sistemas em cursos de pós-graduação, e muito menos nos cursos de graduação. A dependência temporal desses sistemas é deixada em segundo plano, mesmo já fazendo cerca de meio século que um dos primeiros sistemas fora do equilíbrio, a reação de Belousov-Zhabotinsky, ${ }^{1,2}$ tenha sido descoberto. Mais chocante ainda é o fato dessa mesma reação ser utilizada amplamente em demonstrações de química sem que o significado por trás de atrativas mudanças de cor, os processos fora do equilíbrio, seja realmente discutido.

A utilização do equilíbrio termodinâmico como abordagem mais simples aos problemas de interesse acaba nos acomodando, fazendo com que a natural evolução do estudo para sistemas fora do equilíbrio seja, pelo menos até o momento, negligenciada.

O formalismo da Mecânica Estatística, apesar de ser comumente tratado para processos de equilíbrio, também pode ser utilizado para descrever processos fora do equilíbrio. Sabemos que no equilíbrio é necessário se obter a função de partição, grandeza responsável por descrever as propriedades do sistema em estudo. Fundamentalmente, essas funções de partição dependem das probabilidades de ocupação dos estados de um determinado sistema. Ao se estudar sistemas fora do equilíbrio, essas probabilidades de ocupação variam no tempo, assim como as propriedades do sistema. Não basta, então, somente conhecer a probabilidade de ocupação dos estados no equilíbrio, é necessário acompanhar a evolução temporal dessas probabilidades.

A solução para esta questão é descrever as probabilidades associadas a um sistema através de sua dependência temporal. Para tanto, diversos métodos e técnicas foram desenvolvidos. Dentre essas

\footnotetext{
*e-mail: tcorrera@iq.usp.br
}

técnicas, discutidas anteriormente na literatura, ${ }^{3}$ se encontram as equações de Fokker-Planck, equações de Langevin, Equações-Mestras, entre outras. As Equações-Mestras, objetos do presente artigo, serão discutidas devido à possibilidade de serem usadas em diversas áreas do conhecimento, inclusive na Química.

Um exemplo de aplicabilidade das Equações-Mestras que nos é palpável é no campo da Cinética Química. Como se sabe, há diversos métodos matemáticos para descrever a dinâmica de reações químicas. Um deles é o famoso método determinístico das equações de velocidade de reação, com o qual os estudantes estão habituados a lidar em problemas de Cinética Química. No entanto, este método só é preciso quando o número de moléculas é muito elevado, permitindo um tratamento através de um ponto de vista contínuo, pressuposto a partir do uso de concentrações nas equações de velocidade. Quando existem cerca de dezenas ou centenas de moléculas, a aleatoriedade do sistema não pode ser ignorada, exigindo que métodos estocásticos, como a modelagem por Equações-Mestras, sejam utilizados.

Apesar da literatura mais fundamental a respeito de EquaçõesMestras se restringir basicamente a livros e artigos científicos nos campos da Matemática e da Física, muitas aplicações das EquaçõesMestras podem ser encontradas na literatura. Destacam-se, como exemplos, a modelagem de catálise enzimática, ${ }^{4}$ mecanismos de controle do relógio biológico de organismos, ${ }^{5}$ cinética de difusão de átomos em ligas metálicas fora do equilíbrio, ${ }^{6}$ transporte de solutos em meios porosos, ${ }^{7}$ produtos de combustão e reações radicalares, ${ }^{8,9}$ estudo da composição de fase de agregados atômicos, ${ }^{10}$ dinâmica de corpos em aceleradores de partículas, ${ }^{11}$ entre muitas outras. Como vemos, as aplicações das Equações-Mestras podem ser encontradas em todos os ramos da ciência, desde a Biologia à Física elementar, sendo a Química uma das áreas que atualmente mais faz uso das Equações-Mestras para resolver problemas de sistemas fora do equilíbrio.

Em virtude da crescente importância desses sistemas no dia-a-dia do químico, é vital se conhecer os fundamentos e os conceitos físicos por detrás das formulações matemáticas deste método de resolução. Este artigo busca apresentar ao leitor químico tais aspectos. Ao se familiarizar com as Equações-Mestras, alunos de graduação podem reconhecer as limitações de alguns modelos ensinados nos cursos de Química para tratar de sistemas muito pequenos ou de poucos 
corpos, enquanto alunos de pós-graduação e pesquisadores podem reconhecer nas Equações-Mestras um método viável para estudar seus problemas de interesse.

Para explorar as características dessas equações, o presente artigo é dividido de forma a conceituar e deduzir as chamadas EquaçõesMestras, discutir algumas de suas propriedades mais relevantes, exemplificar sua utilização com um simples sistema que possibilite compreender os conceitos físicos envolvidos e, por fim, ilustrar aplicações nas quais esta modelagem é utilizada, como reações químicas de múltiplos poços e a dissociação de íons induzida por radiação térmica.

\section{A EQUAÇÃO-MESTRA}

\section{Qual o significado da Equação-Mestra?}

De modo geral, quando nos referimos à Equação-Mestra, estamos mencionando um conjunto de equações que descrevem a evolução temporal de probabilidades de um determinado sistema.

Partindo para uma definição matemática mais específica, as chamadas Equações-Mestras são equações equivalentes às equações de Chapman-Kolmogorov ${ }^{12,13}$ para processos markovianos, com a vantagem de serem de mais fácil manipulação e mais próximas aos conceitos físicos envolvidos. Resumidamente, equações de Chapman-Kolmogorov descrevem a distribuição de probabilidades em um sistema qualquer, tendo aplicações mais amplas ao custo de uma forma mais hermética. Por sua vez, processos markovianos são processos nos quais as probabilidades futuras são aleatórias e sem memória, ou seja, um sistema onde a probabilidade de um determinado estado futuro depende somente do estado atual e não de qualquer outro estado anterior. ${ }^{14}$

Dessa forma, se conhecermos a evolução temporal de probabilidades de ocupação dos estados de um sistema em função do tempo, podemos determinar como quaisquer propriedades médias desse sistema variam em função do tempo. Isso é possível, pois uma propriedade média $\langle x\rangle$ qualquer pode ser obtida pela Mecânica Estatística através da relação $\langle x\rangle=\sum_{i} p_{i} x_{i}$ onde $p_{i}$ é a probabilidade de ocupação de um estado $i$ qualquer e $x_{i}$ uma dada propriedade tomada para o mesmo estado $i$. Cabe lembrar que podemos calcular qualquer propriedade $\langle x\rangle$, mas isso só terá sentido se esta propriedade estiver definida no instante para o qual foi calculada. Ou seja, se utilizarmos esse cálculo para um instante fora do equilíbrio, a propriedade $\langle x\rangle$ deve estar definida fora do equilíbrio.

O intuito da Equação-Mestra é, portanto, determinar a evolução temporal de um conjunto de probabilidades de ocupação dos estados do sistema. Como as transições de população entre estados influenciam estas probabilidades de ocupação, é necessário determiná-las por equações acopladas, o que será visto mais adiante. Através da descrição dessas probabilidades, é possível modelar sistemas fora do equilíbrio, o que, em última análise, é o objetivo destas equações.

Na próxima seção será ilustrado um modo de se obter uma expressão para a Equação-Mestra. Apesar desta dedução não ser genérica como outras encontradas na literatura, ${ }^{13,15,16}$ ela deixa claro o conceito existente por trás dessa modelagem.

\section{Obtendo a Equação-Mestra}

Suponha um sistema que possua $k$ estados de configuração, em que estes estados podem representar posições, energias, cores, ou quaisquer condições específicas do sistema.

Queremos calcular a quantidade de elementos $N_{l}(t)$ que podem ser encontrados em um estado $l$ qualquer, em um instante $t$ qualquer. Considerando que o sistema é sem memória, podemos calcular a variação do número de ocupação de um determinado estado $l$,
$\Delta N_{l}$, entre o instante $t$ presente e um instante futuro $t+\Delta t$, com $\Delta t$ suficientemente pequeno. Tal variação corresponde ao número de elementos que partem de qualquer estado e chegam ao estado de interesse descontados do número de elementos que deixam o estado para se tornarem parte de qualquer outro estado do sistema, ou seja:

$\Delta N_{l}(t)=N_{l}(t+\Delta t)-N_{l}(t)=\left\{\Delta P_{l, 1}(t) N_{1}(t)+\Delta P_{l, 2}(t) N_{2}(t)+\ldots+\Delta P_{l, k}(t) N_{k}(t)\right\}$

$-\left\{\Delta P_{1, l}(t) N_{l}(t)+\Delta P_{2, l}(t) N_{l}(t)+\ldots+\Delta P_{k, l}(t) N_{l}(t)\right\}$

onde $l$ é um estado qualquer entre $(1,2, \ldots, k), P_{i, j}(t)=P_{i \leftarrow j}(t), i=(1$, $2, \ldots, k), j=(1,2, \ldots, k)$ representa a probabilidade infinitesimal de que ocorra transição para um elemento ir do estado $j$ para o estado $i$ no intervalo de tempo $\Delta t$ e $N_{i}(t)$ representa o número de ocupação do $i$-ésimo estado do sistema em um instante $t$ qualquer.

Na Equação 1, o primeiro termo entre chaves representa o número de elementos que "passam" para o estado $l$, enquanto o segundo termo entre chaves representa o número de elementos que "partem" deste estado $l$ para quaisquer outros estados do sistema. Assim, por exemplo, a quantidade $\Delta P_{l, 1}(t) N_{1}(t)$ corresponde ao acréscimo infinitesimal durante o intervalo de tempo $\Delta t$ no número de ocupação do estado $l$, levando em consideração apenas as transições que partiram do estado 1 . O acréscimo infinitesimal total no número de ocupação do estado $l$, ocorrido no intervalo $\Delta t$, é dado então pela soma de todos os termos que estão dentro da primeira chave no lado direito da Equação 1. Do mesmo modo, a soma dos termos dentro da segunda chave do lado direito da Equação 1 dá o decréscimo infinitesimal total no número de ocupação do estado $l$, ocorrido no intervalo $\Delta t$.

Dividindo a Equação 1 pelo número total de elementos $N$, onde $N=\sum_{i}^{k} N_{i}$ e considerando a variação da probabilidade de ocupação $\Delta P_{l}$ como $\Delta P_{l}=\Delta N_{l} / N$ em um intervalo de tempo $\Delta t$ suficientemente curto, temos:

$$
\begin{gathered}
\Delta P_{l}(t)=\left(\Delta P_{l, 1}(t) P_{1}(t)+\Delta P_{l, 2}(t) P_{2}(t)+\ldots+\Delta P_{l, k}(t) P_{k}(t)\right)- \\
\left(\Delta P_{1, l}(t) P_{l}(t)+\Delta \mathrm{P}_{2, l}(t) P_{l}(t)+\ldots+\Delta P_{k, l}(t) P_{1}(t)\right)
\end{gathered}
$$

Como pode ser observado pela Equação 2, as probabilidades de ocupação são dependentes entre si e, portanto, as equações não podem ser resolvidas separadamente. É necessário se resolver simultaneamente o conjunto de $l$ equações $\Delta P_{l} \operatorname{com} l=(1,2, \ldots, k)$. Isso pode ser representado através de uma notação matricial (neste texto se utilizam letras em negrito para representar grandezas matriciais):

$$
\begin{gathered}
\Delta \boldsymbol{P}=\left(\begin{array}{c}
\Delta P_{1} \\
\Delta P_{2} \\
\Delta P_{3} \\
\vdots \\
\Delta P_{k}
\end{array}\right)=\left(\begin{array}{c}
\Delta P_{1,1} P_{1}+\Delta P_{1,2} P_{2}+\cdots+\Delta P_{1, k} P_{k} \\
\Delta P_{2,1} P_{1}+\Delta P_{2,2} P_{2}+\cdots+\Delta P_{2, k} P_{k} \\
\Delta P_{3,1} P_{1}+\Delta P_{3,2} P_{2}+\cdots+\Delta P_{3, k} P_{k} \\
\vdots \\
\Delta P_{k, 1} P_{1}+\Delta P_{k, 2} P_{2}+\cdots+\Delta P_{k, k} P_{k}
\end{array}\right)- \\
\left(\begin{array}{c}
\Delta P_{1,1} P_{1}+\Delta P_{2,1} P_{1}+\cdots+\Delta P_{k, 1} P_{1} \\
\Delta P_{1,2} P_{2}+\Delta P_{2,2} P_{2}+\cdots+\Delta P_{k, 2} P_{2} \\
\Delta P_{1,3} P_{3}+\Delta P_{2,3} P_{3}+\cdots+\Delta P_{k, 3} P_{3} \\
\vdots \\
\Delta P_{1, k} P_{k}+\Delta P_{2, k} P_{k}+\cdots+\Delta P_{k, k} P_{k}
\end{array}\right)
\end{gathered}
$$

onde as grandezas $\Delta P_{i, j}=\Delta P_{i, j}(t)$ e $P_{l}=P_{l}(t)$ para facilitar a notação. Trabalhando com estas matrizes, podemos reescrevê-las como um produto de matrizes da seguinte forma:

$$
\boldsymbol{\Delta P}=\left(\begin{array}{cccc}
\Delta P_{1,1} & \Delta P_{1,2} & \cdots & \Delta P_{1, k} \\
\Delta P_{2,1} & \Delta P_{2,2} & \cdots & \Delta P_{2, k} \\
\Delta P_{3,1} & \Delta P_{3,2} & \cdots & \Delta P_{3, k} \\
\vdots & \vdots & & \vdots \\
\Delta P_{k, 1} & \Delta P_{k, 2} & \cdots & \Delta P_{k, k}
\end{array}\right)\left(\begin{array}{c}
P_{1} \\
P_{2} \\
P_{3} \\
\vdots \\
P_{k}
\end{array}\right)-
$$




$$
\left(\begin{array}{ccccc}
P_{1} & 0 & 0 & \cdots & 0 \\
0 & P_{2} & 0 & \cdots & 0 \\
0 & 0 & P_{3} & \cdots & 0 \\
\vdots & \vdots & \vdots & \ddots & \vdots \\
0 & 0 & \cdots & 0 & P_{k}
\end{array}\right)\left(\begin{array}{cccc}
\Delta P_{1,1} & \Delta P_{2,1} & \cdots & \Delta P_{k, 1} \\
\Delta P_{1,2} & \Delta P_{2,2} & \cdots & \Delta P_{k, 2} \\
\Delta P_{1,3} & \Delta P_{2,3} & \cdots & \Delta P_{k, 3} \\
\vdots & \vdots & & \vdots \\
\Delta P_{1, k} & \Delta P_{2, k} & \cdots & \Delta P_{k, k}
\end{array}\right)\left(\begin{array}{c}
1 \\
1 \\
1 \\
\vdots \\
1
\end{array}\right)
$$

A operação acima somente separou as matrizes da Equação 3 em um produto de matrizes. No primeiro termo do lado direito, a matriz foi separada em uma matriz que descreve as probabilidades de transição, e outra que descreve as probabilidades de ocupação, assim como no termo negativo, com exceção da matriz unitária, necessária para que a multiplicação descrita na Equação 4 seja equivalente à Equação 3.

Definindo a matriz de probabilidades de transição infinitesimais $\boldsymbol{T}$ como

$$
\boldsymbol{T}=\left(\begin{array}{cccc}
\Delta P_{1,1} & \Delta P_{1,2} & \cdots & \Delta P_{1, k} \\
\Delta P_{2,1} & \Delta P_{2,2} & \cdots & \Delta P_{2, k} \\
\Delta P_{3,1} & \Delta P_{3,2} & \cdots & \Delta P_{3, k} \\
\vdots & \vdots & & \vdots \\
\Delta P_{k, 1} & \Delta P_{k, 2} & \cdots & \Delta P_{k, k}
\end{array}\right)
$$

pode-se representar a Equação 4 na forma da soma abaixo para um determinado estado de interesse $l$ qualquer:

$$
\Delta P_{l}=\sum_{m}\left(\Delta T_{l, m} P_{m}-\Delta T_{m, l} P_{l}\right)
$$

onde o índice da somatória $m=(1,2, \ldots, l, \ldots, k)$ e $\Delta T_{l, m}$ representa o termo da $l$-ésima linha e $m$-ésima coluna da matriz $\boldsymbol{T}$. Assim, a variação da probabilidade de ocupação para um determinado estado $l$ qualquer é dada pela transição de qualquer um dos estados do sistema para o estado $l\left(\sum_{m}\left(\Delta T_{l, m} P_{m}\right)\right)$ descontado de todos os elementos que partem do estado $l$ para qualquer estado $m\left(\sum_{m}\left(\Delta T_{m, l} P_{l}\right)\right)$, em um dado intervalo de tempo infinitesimal $\Delta t$.

A Equação 5 é chamada de Equação de Markov, ${ }^{16}$ por ser a equação que relaciona a probabilidade futura com a probabilidade atual através de uma matriz de probabilidades de transição entre estados.

Como estamos interessados em descrever a evolução temporal das probabilidades de ocupação de um determinado sistema a partir da Equação 5, que é uma diferença discreta entre os momentos $t$ e $t+\Delta t$, podemos reescrevê-la na forma diferencial, ou seja, para $\Delta t \rightarrow 0$, obtendo assim a equação Equação-Mestra ${ }^{12,13,17}$

$$
\frac{d P_{l}}{d t}=\sum_{m}\left(W_{l, m} P_{m}-W_{m, l} P_{l}\right),
$$

onde $W_{l, m}$ representa o termo da $l$-ésima linha e $m$-ésima coluna da matriz $\boldsymbol{W}$, chamada matriz de coeficientes de transição, dada por:

$$
\boldsymbol{W}=\left(\begin{array}{cccc}
R_{1,1} & R_{1,2} & \cdots & R_{1, k} \\
R_{2,1} & R_{2,2} & \cdots & R_{2, k} \\
R_{3,1} & R_{3,2} & \cdots & R_{3, k} \\
\vdots & \vdots & & \vdots \\
R_{k, 1} & R_{k, 2} & \cdots & R_{k, k}
\end{array}\right)
$$

onde $R_{i, j}=d P_{i, j} / d t$. Cabe ressaltar que, como $P_{i, j}$ é uma probabilidade, a unidade de $R_{i, j}$ é [unidade de tempo] ${ }^{-1}$, ou seja, $R_{i, j}$ é uma frequência de transição entre os estados $j$ e $i$.

A Equação 6, chamada Equação-Mestra, descreve a evolução da probabilidade de ocupação dos estados de um determinado sistema. Neste ponto cabe dizer que a nomenclatura "Equação-Mestra" não é a mais recomendada por não ser específica o suficiente. Essa nomenclatura vem do seu primeiro uso na literatura, ${ }^{18}$ no qual essa equação foi utilizada para deduzir uma série de outras equações e, portanto, foi denominada "Mestra", ou seja, aquela que guia e conduz. A nomenclatura mais recomendada para uma equação que relacione mudanças de probabilidades em função do tempo seria "equações-M", seguindo sugestão de M. Kac. ${ }^{12}$ Cabe ressaltar que, dependendo das considerações feitas na dedução de Equações-Mestras, podemos obter variações da Equação 6. Um nome mais específico para a equação deduzida aqui seria "Equação-Mestra de Pauli", em referência a uma equação de mesma forma, deduzida por Pauli.

\section{Algumas propriedades das Equações-Mestras}

A forma da Equação 6 nos permite facilmente analisar seu significado. O primeiro termo, positivo, descreve todos os termos $m$ que dão origem a termos $l$, e é conhecido como "termo de ganho". Da mesma forma, o segundo termo, negativo, é chamado de "termo de perda", justamente por relacionar a saída de termos de $l$ para qualquer outro estado do sistema. A mesma observação pode ser feita de maneira mais direta a partir da Equação 2, usada para a dedução da Equação-Mestra. Desta forma, a Equação-Mestra é uma equação de ganho e perda para as probabilidades de ocupação dos estados do sistema ao longo do tempo.

Além disso, cabe ressaltar que esta equação pode também ser escrita na forma contínua ao invés de ser construída para estados discretos, possibilitando uma maior abrangência de sistemas modelados. Entretanto, em algumas aplicações em que a forma contínua da Equação-Mestra poderia ser usada, certo intervalo de estados contínuos de características próximas são agrupados, causando uma discretização do sistema contínuo.

Um exemplo destes estados contínuos seria, por exemplo, na análise de cores. Se podem existir todas as cores da escala entre o vermelho e o violeta, e definirmos um estado para cada uma dessas cores, teremos um sistema contínuo. Entretanto, estados que possuam as mesmas características, ou seja, cores muito próximas, podem ser considerados como uma só cor. Dessa forma, todo o nosso espectro de cores pode ser resumido em 8, 16, 32 ou, por exemplo, 256 milhões de cores, como fazem algumas placas de vídeo de computadores.

Cabe lembrar também, que por ser deduzida diretamente de uma equação de Markov, ou seja, por descrever processos sem memória, a Equação-Mestra segue as propriedades das equações de Markov. ${ }^{19}$

Uma dessas propriedades que nos é relevante é o comportamento limite dos vetores-estado de uma equação de Markov. Esse comportamento pode ser descrito da seguinte forma: Se o tempo de um processo de Markov tende para o infinito, $t \rightarrow \infty$, o autovetor $\mathbf{P}$ converge se, e somente se, a matriz $\mathbf{T}$ e, consequentemente, $\mathbf{W}$, forem matrizes regulares, ou seja, se tiverem todas as suas entradas positivas, ou se existir qualquer potência $\mathrm{s},(\mathrm{s} \in$ números naturais), tal que $(\mathbf{T})^{\mathrm{s}}$ tenha todas as suas entradas positivas.

Esse teorema, cuja dedução pode ser encontrada na literatura, ${ }^{20} \mathrm{se}$ reflete na Equação-Mestra. Quando $t \rightarrow \infty$, o autovetor $\boldsymbol{P}$ converge, $\boldsymbol{P} \rightarrow \boldsymbol{P}_{e q}$, onde $\boldsymbol{P}_{e q}$ representa o vetor de probabilidades de ocupação do sistema quando este está no equilíbrio, ou seja, quando não há mais troca líquida entre os estados do sistema. ${ }^{17}$ Isso quer dizer que se a matriz $\boldsymbol{W}$ é regular, o que ocorre na maioria das aplicações, pois as probabilidades são positivas, o sistema tende ao equilíbrio, e a Equação-Mestra é capaz de descrevê-lo até esse instante.

Apesar desta imposição restringir o uso da Equação-Mestra, veremos que a maioria dos sistemas de interesse caminha para o equilíbrio e, portanto, possuem matrizes de coeficientes de transição regulares. Esses sistemas são criados por perturbações pequenas no equilíbrio e, por isso, tendem a retornar a essa condição. Isso se deve 
ao fato de que tais fenômenos se encontram no regime linear, onde pequenas perturbações do equilíbrio não são suficientes para dirigir o sistema por bifurcações que o distanciariam do equilíbrio. Neste regime linear são válidas as leis de Onsager e o comportamento do corpo é uma extensão linear do seu comportamento no estado de equilíbrio. Ademais, cabe dizer que, quando a matriz $\boldsymbol{W}$ puder ser fatorada em mais de uma matriz, esse sistema converge para uma das possíveis configurações de equilíbrio.

Outra característica que deve ser ressaltada é que a EquaçãoMestra exibe, para um sistema fechado e no equilíbrio, balanço detalhado ${ }^{12,17}$ para cada par de estados do sistema. Ou seja, no equilíbrio, o que parte de um estado $i$ em direção a um estado $j$ é igual ao que parte desse estado $j$ em direção ao estado $i$, como mostra a Equação 7:

$$
W_{j, i} P_{i_{e q}}=W_{i, j} P_{j e q}
$$

Como vemos, essas propriedades são as mesmas que encontramos para processos químicos corriqueiros, o que faz a modelagem por Equação-Mestra ser adequada para estes processos.

A seguir, iremos trabalhar um exemplo ilustrando o processo de decaimento radioativo. Apesar de simplificado, esse exemplo ajuda a ilustrar os conceitos envolvidos na utilização da Equação-Mestra, além de permitir comparação direta com o problema físico modelado.

\section{Decaimento radioativo}

O objetivo desta seção é aplicar a modelagem por Equação-Mestra a um sistema simples, o decaimento radioativo de um elemento químico, como sugerido na literatura. ${ }^{13}$ Tomando, por exemplo, o decaimento de ${ }^{235} \mathrm{U}$ (representado adiante somente como $\mathrm{U}$ ) a ${ }^{231} \mathrm{Th}$ (representado adiante somente como Th), iremos considerar um sistema de dois estados, o estado "Urânio" representado por $U$ e o estado "Tório" representado por $T$. Entende-se, nesse contexto, que $N_{x}(t)$, o número de ocupação do estado $X$, corresponde ao número de átomos de $\mathrm{X}$ presentes na amostra em um determinado instante qualquer.

Vamos considerar também os coeficientes de transição entre os esta$\operatorname{dos} U$ e $T, R_{T, U}=R_{U \rightarrow T}=\kappa$, a taxa (ou frequência) de decaimento radioativo. Nesse caso, todos os outros coeficientes são tomados como zero, já que átomos de U não geram novos átomos de $\mathrm{U}\left(R_{U, U}=R_{U \rightarrow U}=0\right)$, átomos de Th não geram novos átomos de Th $\left(R_{T T}=R_{T \rightarrow T}=0\right) \mathrm{e}$ não ocorre a formação de U a partir de Th $\left(R_{U, T}=R_{T \rightarrow U}=0\right)$. Dessa forma, a Equação-Mestra (6) para o sistema pode ser escrita como $d P_{l} / d t=\sum_{m}\left(W_{l m} P_{m}-W_{m l} P_{l}\right)$. Assim, temos:

$$
\frac{d \boldsymbol{P}}{d t}=\frac{d}{d t}\left(\begin{array}{c}
P_{U} \\
P_{T}
\end{array}\right)=\left(\begin{array}{l}
P_{U} R_{U, U}+P_{T} R_{U, T} \\
P_{U} R_{T, U}+P_{T} R_{T, T}
\end{array}\right)-\left(\begin{array}{c}
P_{U} R_{U, U}+P_{U} R_{T, U} \\
P_{T} R_{U, T}+P_{T} R_{T, T}
\end{array}\right)
$$

Substituindo os valores dos coeficientes de transferência, temos:

$$
\frac{d \boldsymbol{P}}{d t}=\frac{d}{d t}\left(\begin{array}{c}
P_{U} \\
P_{T}
\end{array}\right)=\left(\begin{array}{c}
0+0 \\
\kappa P_{U}+0
\end{array}\right)-\left(\begin{array}{c}
0+\kappa P_{U} \\
0+0
\end{array}\right)
$$

Para resolvermos essa equação, podemos escrever a equação matricial como duas equações diferencias acopladas:

$$
\left.\begin{array}{l}
\frac{d P_{U}}{d t}=-\kappa P_{U} \\
\frac{d P_{T}}{d t}=+\kappa P_{U}
\end{array}\right\}
$$

Integrando a primeira equação e considerando que no início só existem elementos no estado "Urânio" $\left(P_{U}(0)=1\right.$ e $\left.N_{U}(0)=N\right)$ temos:

$$
\begin{gathered}
P_{U}(t)=e^{-\kappa t} \\
N_{U}(t)=N_{U}(0) e^{-\kappa t}
\end{gathered}
$$

O resultado da Equação 10 é exatamente o que se espera para um processo de decaimento radioativo, onde $N_{U}(0)$ é o número de elementos no estado "Urânio" no tempo $t=0$.

Esse exemplo simples ilustra três pontos importantes. Primeiro, vemos que a física do problema é definida pela matriz $\boldsymbol{W}$, e que é alterando os coeficientes de transporte que podemos modelar sistemas diferentes. Nesse sentido, podemos facilmente modelar, por exemplo, elementos de Th regenerando $\mathrm{U}$ simplesmente tornando o coeficiente de transição de "Tório" para "Urânio" $\mathrm{R}_{T \rightarrow U}>0$. Cabe ressaltar que, neste caso, reações sucessivas podem também ser modeladas, bastando aumentar o número de estados e definindo $\boldsymbol{W}$ de maneira conveniente.

Segundo, vemos pelas Equações 9 que o sistema exibe balanço detalhado, já que a taxa de consumo de $\mathrm{U},\left|d P_{U} / d t\right|$ é igual à taxa de formação de Th, $\left|d P_{T} / d t\right|$. Isso é reflexo dos princípios fundamentais da Química, que afirmam que a matéria não pode ser criada ou destruída.

Por fim, podemos ver que se a matriz de coeficientes de transição não for suficientemente simples, a solução se tornará mais trabalhosa. Em muitos casos, a complexidade é tão elevada que é impossível escrever uma solução analítica. Entretanto, se o mesmo motivo não é suficiente para invalidar o uso da Mecânica Quântica, também não o será para este caso.

Assim, a próxima seção será dedicada a métodos de resolução das Equações-Mestras, principalmente aos métodos de resolução numéricos.

\section{Resolvendo a Equação-Mestra}

Apesar da Equação-Mestra poder ser resolvida analiticamente para alguns sistemas simples, que possuem condições de contorno bem definidas, ${ }^{15}$ pequenas alterações no sistema modelado podem impossibilitar sua resolução.

Suponha, por exemplo, ${ }^{16}$ que desejamos modelar uma reação química em um sistema, onde existam 100 moléculas que podem sofrer reações, gerando 2 novas espécies. Sendo assim, cada uma das 100 moléculas pode ocupar 1 dos 3 estados do sistema (reagente, produto 1 e produto 2 ), gerando um total de $100^{3}$ estados e, consequentemente, 1.000.000 equações diferenciais acopladas! Nesse caso específico, esse sistema pode ser resolvido para cada concentração de espécies, gerando 3 equações acopladas. Porém, de forma geral, apenas para um resumido grupo de aplicações são obtidas soluções analíticas para as Equações-Mestras. Nos outros casos, é necessária a utilização de métodos de resolução numéricos, ou outras abordagens para a sua resolução, tais como, diagonalização da matriz de coeficientes de transição $\boldsymbol{W}$, expansão da Equação-Mestra em série de potências, entre outros.

Apesar de serem comumente utilizados, tais métodos não serão aqui descritos por não serem tão próximos aos conceitos fundamentais quanto os métodos numéricos, descritos a seguir. Mais informações a respeito de outros métodos de resolução podem ser encontradas na literatura. ${ }^{12,13,15}$

Dentre os métodos numéricos, diversas abordagens já foram criadas, cada qual mais específica e eficiente para um problema particular a ser modelado. Portanto, somente uma explicação geral do funcionamento desses algoritmos numéricos será realizada.

Um programa típico para a resolução da Equação-Mestra consiste, em um primeiro passo, em assumir uma condição inicial para o vetor de probabilidade $\boldsymbol{P}(n), n=0$ e de posse da matriz de coeficientes de 
transição $\boldsymbol{W}$, obter o novo vetor $\boldsymbol{P}(n+1)$, que é tomado como início da próxima etapa (Figura 1).

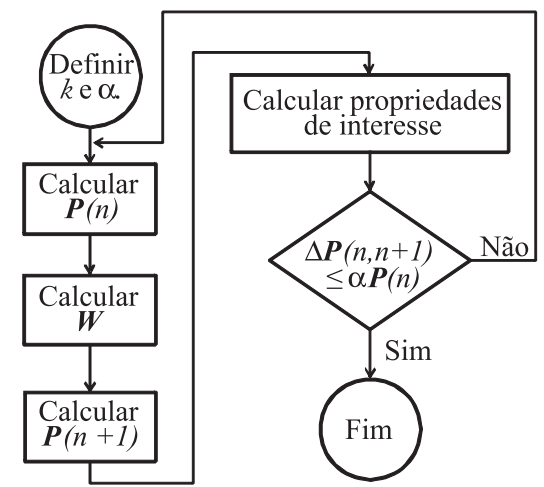

Figura 1. Diagrama de blocos genérico de um programa para resolução da Equação-Mestra. O parâmetro $k$ representa o número de estados, definido por suas propriedades; $n$ um passo da simulação; $\boldsymbol{W}$ a matriz de coeficientes de transição; $\boldsymbol{P}(n)$ o vetor de probabilidades de ocupação em um passo $n$, enquanto $\alpha$, chamado de parâmetro de convergência, representa o parâmetro responsável pelo fim do cálculo

A determinação da distribuição inicial é feita considerando-se os níveis energéticos do sistema, mas de forma que esta distribuição seja fora do equilíbrio.

A matriz de coeficientes de transição $\boldsymbol{W}$ é o que define o problema físico a ser estudado. Em alguns casos, esta matriz depende de propriedades do sistema como, por exemplo, a energia total. Por este motivo, essa matriz deve ser recalculada a cada passo do cálculo.

Após a determinação de um novo valor para o vetor de probabilidades de ocupação, propriedades médias $\langle x\rangle$ de interesse do sistema podem ser calculadas através da relação $\langle x\rangle=\Sigma_{i} p_{i} x_{i}$. Quaisquer propriedades poderão ser calculadas, entretanto apenas aquelas definidas fora do equilíbrio é que terão significado físico.

Como a Equação-Mestra leva os processos ao equilíbrio, o cálculo é finalizado quando os vetores de probabilidade de dois passos consecutivos forem iguais, dentro de um intervalo arbitrário de tolerância definido pelo parâmetro de convergência $\alpha$ (Figura 1).

É inerente ao método descrito acima que o tempo seja considerado como discreto ${ }^{21} \mathrm{e}$ evolua em passos $\Delta t$. Entretanto, esse procedimento não representa problema, já que no limite de $\Delta t \rightarrow 0$ (ou seja, $\Delta t$ pequeno o suficiente para a escala de tempo do processo estudado) os passos de tempo tendem a um comportamento contínuo.

Cabe ressaltar que dependendo do processo modelado, estes passos $\Delta t$ podem assumir ordens de grandezas distintas. Supondo, por exemplo, a simulação de um processo no nível molecular, como reações químicas, passos extremamente pequenos, da ordem de ps, serão necessários. Entretanto, no caso do decaimento radioativo, cuja meia vida pode ser expressa em anos, passos da ordem de grandeza de um mês são perfeitamente plausíveis. Portanto, é necessário escolher passos coerentes com os fenômenos modelados.

Como para cada iteração são obtidos os valores das propriedades médias do sistema, pode-se descrever a evolução dessas propriedades até que o sistema atinja o equilíbrio, bem como seus valores no estado de equilíbrio.

O procedimento acima é genérico e tem como objetivo ilustrar os passos por trás de uma simulação para resolução da Equação-Mestra. Como cada aplicação tem um modo peculiar de ser tratada, a próxima seção, além de ilustrar aplicações mais específicas, mostra como os problemas são modelados pela Equação-Mestra e como podemos trabalhar com a matriz de coeficientes de transição $\boldsymbol{W}$ e a distribuição inicial de probabilidades $\boldsymbol{P}(0)$ para incorporarmos nossas condições de contorno no modelo de interesse.

\section{Aplicações}

Esta seção descreve duas aplicações das Equações-Mestras encontradas na literatura. Serão mostradas algumas considerações necessárias para modelar os problemas de interesse, bem como os resultados obtidos através de sua utilização. O primeiro caso trata da modelagem de uma reação de combinação de radicais, enquanto o segundo estuda reações induzidas por radiação térmica.

No caso do primeiro exemplo, reações entre radicais são estudadas via Equações-Mestras. Tipicamente, radicais apresentam-se em baixa concentração em meios reacionais, inviabilizando um estudo acurado através das equações de velocidade. Já em uma abordagem por Equações-Mestras, cada um dos diferentes produtos para os quais os reagentes radicalares podem evoluir representa um estado distinto, cada qual com uma energia diferente e, portanto, uma probabilidade de ocupação diferente, que muda com o tempo. Tal exemplo é representativo de uma grande classe de problemas cinéticos que podem ser tratados com a Equação-Mestra.

O caso do segundo exemplo é ainda mais fundamental e evidencia a larga aplicabilidade das Equações-Mestras. Processos de absorção ou emissão de energia podem gerar estados eletrônicos, vibracionais ou rotacionais excitados. Tais espécies excitadas podem reagir de maneira peculiar, levando a uma miríade de produtos bastante vasta. Assim, modelar a distribuição de espécies entre seus possíveis estados é importante para se predizer a reatividade de um sistema químico. Nesse sentido, mais uma vez, as Equações-Mestras se mostram um método estocástico poderoso para suprir a limitação dos modelos analíticos contínuos e prover uma alternativa a métodos experimentais complexos de contagem de fótons emitidos ou absorvidos, por exemplo.

\section{Recombinação de radicais propargil}

Miller e Klippenstein, ${ }^{22}$ em 2003, investigaram teoricamente a distribuição de produtos no equilíbrio para a reação de recombinação de radicais propargil $\left(\mathrm{C}_{3} \mathrm{H}_{3}\right)$.

$\mathrm{O}$ interesse dos pesquisadores nesse sistema, assim como em outros radicais, ${ }^{23}$ vem do fato dessas espécies servirem de modelo para os radicais presentes na combustão, que por possuírem um alto tempo de vida, devido à sua estabilização por ressonância, contribuem para a formação de hidrocarbonetos poliaromáticos (HPA) nos processos de queima.

A reação de recombinação de radicais propargil apresenta uma superfície de potencial com múltiplos poços, e a relação entre as velocidades de reação entre esses pontos estacionários determinará, em última análise, a distribuição de produtos no equilíbrio.

Do ponto de vista da Equação-Mestra, esse sistema é descrito da seguinte forma: a) define-se um estado para cada ponto definido pelos fundos dos poços da superfície de energia potencial; b) as taxas de transição $R_{i, j}$ entre estados são tomadas como a velocidade de reação entre os poços, dadas pelas constantes de velocidade microcanônica, obtidas, por sua vez, pela teoria de reatividade química de RiceRamsperger-Kassel-Markus (RRKM). ${ }^{24}$

Para fins didáticos, a Figura 2 ilustra como são considerados os estados e as taxas de transição para uma reação de múltiplos poços hipotética, análoga à analisada por Miller e Klippenstein. ${ }^{22}$

Pode-se observar pela matriz $\boldsymbol{W}$ da Figura 2 que as constantes de velocidade serão zero para estados não ligados por um estado de transição. Por exemplo, o elemento $W_{3,1}$ da matriz representa a velocidade da reação $\mathrm{R} \rightarrow 2$, que, pela figura, deve ser zero, já que 


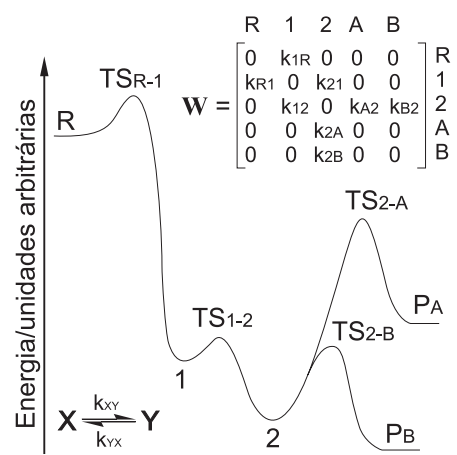

Figura 2. Superfície de energia potencial ao longo da coordenada de reação para uma reação de múltiplos poços hipotética. $R, 1,2, P_{A}, P_{B}$ representamo reagente, intermediário 1 , intermediário 2 , produto $A$ e produto $B$, respectivamente. $T S_{X-Y}$ e $k_{X Y}$ representam o estado de transição entre os estados $X$ e $Y$ e a constante de velocidade para a reação $X \rightarrow Y$, respectivamente

o estado R nunca poderá gerar o estado 2 diretamente, pois não há nenhum estado de transição que conecte os dois mínimos de energia. No caso do elemento $W_{3,4}$, representando a reação $2 \rightarrow \mathrm{A}$, existe um estado de transição que conecta os dois mínimos, e este elemento corresponderá à constante de velocidade da respectiva reação $\left(\mathrm{k}_{2 \mathrm{~A}}\right)$.

Cabe notar que o estado de transição também conecta os mínimos no caminho reverso ao da coordenada de reação, fazendo com que o elemento $W_{3,4}$, representando a reação inversa $\mathrm{A} \rightarrow 2$, também seja diferente de zero.

Assim, partindo-se de uma distribuição qualquer de componentes do sistema fora do equilíbrio, como somente um dos poços ocupado, por exemplo, a resolução da Equação-Mestra levará o sistema até a distribuição no equilíbrio. Em outras palavras, resolver a EquaçãoMestra para este sistema implica em se partir de um vetor de probabilidades de ocupação qualquer e efetuar transições até que ele convirja para seu valor no equilíbrio.

Como as constantes de velocidade dependem de parâmetros como pressão e temperatura, é possível variar estes valores, obtendo-se assim a distribuição de produtos em função destas grandezas.

A partir dos resultados obtidos, os autores observaram para o sistema três regimes de comportamento diferentes em função da temperatura. Primeiro, para valores baixos de temperatura, ou seja, temperaturas abaixo de $800 \mathrm{~K}$, no limite de pressões baixas, onde não ocorre estabilização dos complexos intermediários, os únicos produtos observados são os produtos finais de reação, radical fenila e próton. Ao aumentar a pressão, os poços mais fundos se estabilizam e os complexos intermediários são identificados pela solução da Equação-Mestra.

Para temperaturas acima de $800 \mathrm{~K}$, as colisões entre as espécies reagentes se tornam mais energéticas, de forma que as colisões de ativação e desativação ocorram em extensões comparáveis. Sendo assim, com o aumento de temperatura, os limites de estabilização (temperatura a partir da qual todas as colisões causam ativação) para cada um dos mínimos é ultrapassado, até que a $2000 \mathrm{~K}$ não ocorre mais estabilização de nenhum dos complexos e somente os produtos finais são observados.

A análise dos autovetores da matriz de coeficientes de transição diagonalizada também é muito instrutiva. Através da análise destes autovetores em função da temperatura é possível determinar qual estado de transição controla a distribuição de produtos na reação. Uma extensa discussão a respeito é feita pelos autores ${ }^{22}$ e não será aqui realizada.

Dessa forma, além do uso da Equação-Mestra possibilitar a obtenção de informações importantes para o estudo de reações, há a vantagem de se poder detectar virtualmente quaisquer produtos, mesmo que estes não possuam características que permitam sua detecção, ou mesmo que estejam em quantidades muito pequenas, condições que impossibilitariam suas determinações experimentais.

\section{Dissociação de agregados iônicos por radiação térmica}

Dunbar e colaboradores ${ }^{25}$ relataram, em 1995, a dissociação de agregados iônicos em experimentos de reatividade em fase gasosa utilizando um espectrômetro de massas por transformada de Fourier. Tal ocorrência foi atribuída à radiação térmica de fundo que emana da cela na qual os agregados são manipulados, conforme a hipótese radioativa de Perrin. ${ }^{26,27}$ Apesar da hipótese de Perrin propor que todas as reações são promovidas por radiação eletromagnética, o que é sabidamente equivocado, tais experimentos de dissociação são provas de que a luz é capaz de promover reações quando interage com a matéria, desde que algumas condições sejam respeitadas. ${ }^{27}$

$\mathrm{O}$ interesse em tal problema não se resume apenas à elucidação da questão levantada, mas também porque o entendimento das reações promovidas por radiação pode fornecer um método eficiente de síntese de íons em fase gasosa, assim como servir, por exemplo, de base teórica para o entendimento da espectroscopia de ação no infravermelho, técnica fundamental de elucidação estrutural acoplada à espectrometria de massas. ${ }^{28}$

Cabe ressaltar que a absorção de radiação pode levar as espécies a estados eletrônicos, vibracionais e rotacionais excitados, mas as faixas de frequência da radiação incidente necessárias para provocar transições eletrônicas, vibracionais ou rotacionais são bastante distintas entre si. Dessa forma, os autores estudam somente as frequências vibracionais, mas todas as outras mudanças poderiam ser modeladas, caso houvesse interesse.

Do ponto de vista molecular, a radiação, quando absorvida pelas moléculas ou íons, é redistribuída estatisticamente nos modos normais de vibração, contribuindo para a energia total da espécie. Durante certo tempo, uma espécie passa por processos sucessivos de ganho e perda de energia vibracional, absorvendo ou emitindo fótons, nos diversos modos normais de vibração. Caso essa espécie absorva energia suficiente para superar a energia crítica $E_{0}$ de reação, irá reagir com uma velocidade dada pela teoria RRKM (Figura 3). ${ }^{24}$

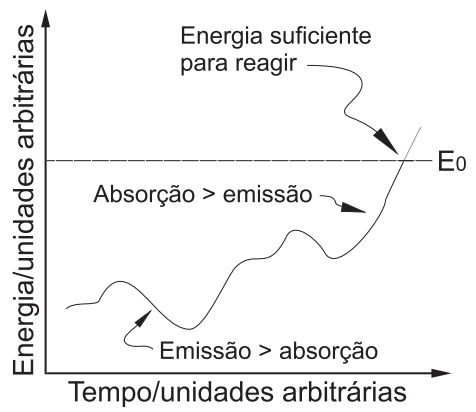

Figura 3. Variação de energia interna de uma espécie capaz de absorver e emitir fótons na região do infravermelho em função do tempo, baseada em Dunbar e McMahon. ${ }^{27}$ Se a taxa de absorção de energia for maior que a de emissão, a espécie ganha energia, podendo atingir a energia crítica de reação $E_{0}$, sendo então susceptível à reação de dissociação

Assim, ao se modelar este problema, considera-se certo número de níveis vibracionais $v$ para cada modo normal de vibração. Cabe destacar que, teoricamente, devem-se considerar infinitos níveis, mas o que se faz é estipular um máximo de energia e agrupar níveis vibracionais com energias próximas e tratá-los como um só estado.

Dado que esses níveis poderão emitir ou absorver fótons no infravermelho (Figura 3), a energia interna total do íon $\langle E\rangle$, dada pela 
relação $\langle E\rangle=\Sigma_{i} E_{i} P_{i}$, sendo $E_{i}$ a energia de cada nível vibracional da espécie, irá variar durante o tempo, sendo justamente o comportamento que se deseja monitorar.

Por sua vez, a matriz de coeficientes de transição será dada, no limite de pressões baixas, ou seja, excluindo os efeitos de colisões, pelos seguintes elementos:

$$
\begin{array}{cc}
R_{i \rightarrow j}=0 & \text { se } i \neq j \pm 1,(1 \\
R_{i \rightarrow j}=\sum_{v} \rho\left(\Delta E_{i j}\right) B_{(v) i \rightarrow j} & \text { se } i=j+1, \\
R_{i \rightarrow j}=\sum_{v} \rho\left(\Delta E_{i j}\right) C_{(v) i \rightarrow j}+\sum_{v} A_{(v) i \rightarrow j} & \text { se } i=j-1,(13)
\end{array}
$$

onde $A_{(v) i \rightarrow j}, B_{(v) i \rightarrow j}$ e $C_{(v) i \rightarrow j}$ são, respectivamente, os coeficientes de emissão espontânea, absorção, emissão induzida de Einstein para cada nível vibracional $v$, e $\rho$, a distribuição de radiação do corpo negro em função da diferença de energia $\Delta E_{i j}$ entre os estados, é dada por:

$$
\rho\left(\Delta E_{i j}\right)=\frac{8 \pi\left(\Delta E_{i j}\right)^{3}}{c^{3} h^{2}}\left(e^{\frac{\Delta E_{i j}}{k T}}-1\right)^{-1}
$$

onde $c$ representa a velocidade da luz no vácuo, $h$ a constante de Planck, $k$ a constante de Boltzmann e $T$ representa a temperatura absoluta na qual estes elementos são calculados.

Novamente, a física do problema estudado é modulada nestes elementos. A condição descrita na Equação 11, por exemplo, obriga que a transferência entre estados não consecutivos a seja zero, de acordo com a regra de seleção $\left(\Delta_{i \rightarrow j}= \pm 1\right)$.

Outro fato importante nesta modelagem é o vetor de probabilidades iniciais $\boldsymbol{P}(0)$. Para se determinar a distribuição inicial de ocupação dos estados do sistema utilizam-se os valores de energia dos níveis vibracionais em uma distribuição de Boltzmann. Entretanto, como para esse sistema consideramos que qualquer espécie com energia total maior que energia crítica reaja, a probabilidade de se encontrar espécies com $\langle E\rangle>E_{0}$ deve ir rapidamente para zero. A distribuição que representa esse comportamento é conhecida como distribuição de Boltzmann truncada. ${ }^{27,29}$

De posse de todos esses parâmetros, é possível resolver a Equação-Mestra. Sua resolução irá fornecer a evolução do vetor de probabilidades no tempo, até que seja atingido o equilíbrio. Em termos físicos, a radiação irá elevar a energia dos íons até que eles possam reagir, sendo, portanto, retirados do sistema. Isso ocorrerá até que mais nenhum íon atinja o limiar de dissociação, ou seja, até que ocorra compensação entre os quanta de energia absorvidos e emitidos pelos níveis vibracionais, de modo a manter a energia média total $\langle E\rangle$ abaixo do limiar de dissociação. Isso fará com que certa proporção de íons produtos e reagentes exista constantemente no sistema, sendo esta exatamente a composição de equilíbrio.

A taxa com que os íons atingem a energia crítica, em conjunto com a velocidade de reação microcânonica, dependente da energia interna do íon, é, por fim, a constante de velocidade de dissociação do íon estudado. Cabe ressaltar que, se a velocidade de reação obtida pela teoria RRKM for muito alta, se pode considerar que todo o íon que atinge a condição $\langle E\rangle>E_{0}$ se transforma imediatamente em produto, aproximação conhecida como "Morte Súbita". ${ }^{30}$ Para o

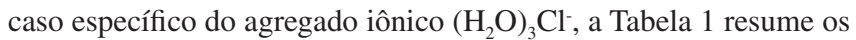
resultados teóricos e experimentais obtidos pelos autores. ${ }^{25}$

Desses resultados é possível se observar uma boa concordância entre teoria e experimento, utilizando apenas a energia crítica como parâmetro ajustável. Vemos, por exemplo, que para o valor de pres-
Tabela 1. Constantes de velocidades experimentais e teóricas obtidas para a dissociação do agregado iônico $\left(\mathrm{H}_{2} \mathrm{O}\right)_{3} \mathrm{Cl}^{-} \cdot{ }^{25} \mathrm{O}$ comportamento das constantes de velocidade frente ao aumento de energia crítica e de temperatura ilustram a coerência do modelo

\begin{tabular}{ccccccccc}
\hline \multicolumn{3}{c}{ Experimental } & & \multicolumn{5}{c}{ Teórico } \\
\cline { 1 - 4 } \cline { 5 - 7 } $\begin{array}{c}\text { Pressão de } \\
\text { fundo (mbar) }\end{array}$ & $\begin{array}{c}k_{300 K} \\
\left(\mathrm{~s}^{-1}\right)\end{array}$ & $\begin{array}{c}E_{a} \\
\left(\mathrm{kcal} \mathrm{mol}^{-1}\right)\end{array}$ & & $\begin{array}{c}E_{0} \text { adotado } \\
\left(\mathrm{kcal} \mathrm{mol}^{-1}\right)\end{array}$ & $\begin{array}{c}k_{300 K} \\
\left(\mathrm{~s}^{-1}\right)\end{array}$ & $\begin{array}{c}k_{330 K} \\
\left(\mathrm{~s}^{-1}\right)\end{array}$ & $\begin{array}{c}E_{a} \\
\left(\mathrm{kcal} \mathrm{mol}^{-1}\right)\end{array}$ \\
\hline $6,610^{-8}$ & 0,122 & 4,8 & & 6,3 & 0,25 & 0,48 & 4,3 \\
$2,510^{-9}$ & 0,11 & 5,0 & & 7,4 & 0,124 & 0,28 & 5,4 \\
& & & 8,6 & 0,057 & 0,15 & 6,4 \\
& & & 9,5 & 0,028 & 0,086 & 7,3 \\
& & & 9,7 & 0,024 & 0,076 & 7,5 \\
& & & 10,8 & 0,0095 & 0,035 & 8,5 \\
\hline
\end{tabular}

são mais baixa $\left(2,510^{-9}\right.$ mbar $)$ e a $300 \mathrm{~K}$, a constante de velocidade experimental é de $0,11 \mathrm{~s}^{-1}$ e a energia de ativação determinada para o processo vale $5,0 \mathrm{kcal} \mathrm{mol}^{-1}$. Para aproximadamente a mesma energia de ativação $\left(5,4 \mathrm{kcal} \mathrm{mol}^{-1}\right)$, a modelagem por Equação-Mestra fornece o valor $0,124 \mathrm{~s}^{-1}$ para a mesma constante de velocidade a $300 \mathrm{~K}$. Cabe ressaltar que, como o modelo foi deduzido para o limite teórico de pressão zero, ou seja, no regime sem colisões, a comparação do experimento realizado a uma pressão mais alta $\left(6,610^{-8} \mathrm{mbar}\right)$ terá um desvio maior do valor previsto teoricamente.

Além disso, pode-se ver que as tendências esperadas para o comportamento frente a aumentos de temperatura e de energia crítica são respeitadas. As constantes de velocidade caem quando são utilizados valores maiores de $E_{0}$ e aumentam quando a temperatura é elevada. Sendo assim, vemos que além de modelar diretamente reações químicas, é possível descrever com o auxílio da Equação-Mestra processos de ganho e perda de energia que, por sua vez, causarão reações químicas.

Cabe ressaltar que através desse modelo os autores são capazes de determinar grandezas relacionadas à absorção de energia. Neste trabalho, por exemplo, foi possível determinar a taxa de absorção e emissão de fótons, o que permite, em última análise, determinar o número de fótons envolvidos no processo de dissociação do íon, medida de difícil determinação experimental.

\section{CONCLUSÃO}

Do que foi discutido, nota-se que, além de possibilitar a modelagem de eventos que tendem ao equilíbrio, a Equação-Mestra é um modo de se introduzir o estudo de processos fora do equilíbrio, devido à sua forte relação com os conceitos físicos, o que não ocorre tão claramente em outras modelagens.

Quanto às aplicações, vemos que a Equação-Mestra é um modelo que pode fornecer informações muito detalhadas e precisas do sistema em estudo, que dificilmente seriam obtidas experimentalmente, permitindo que muitos problemas sejam investigados com elevado grau de profundidade.

Esses motivos explicam o crescente interesse nessa classe de equações e justificam a realização da presente discussão como material de referência na língua portuguesa.

\section{AGRADECIMENTOS}

Ao Prof. J. M. Riveros Nigra pela ajuda na discussão e organização do artigo, ao Sr. Y. A. Aoto pelas proveitosas discussões e aos assessores pelas valiosas modificações sugeridas. Os autores agradecem também ao CNPq (Conselho Nacional de Desenvolvimento Científico e Tecnológico) pelo apoio financeiro. 


\section{REFERÊNCIAS}

1. Belousov, B. P.; Compilation of Abstracts in Radiation Medicine 1959, $147,145$.

2. Zhabotinsky, A. M.; Biofizika 1964, 9, 306.

3. Mazenko, G. F.; Nonequilibrium Statistical Mechanics, $1^{\mathrm{a}}$ ed., WileyVCH GmbH \& Co KGaA: Weinheim, 2006, cap. 1.

4. Das, B.; Gangopadhyay G.; J. Chem. Phys. 2010, 132, 135102-1-8.

5. Vilar, J. M. G.; Kueh, H. Y.; Barkai, N.; Leibler, S.; PNAS 2002, 99, 5988.

6. Vaks, V. G.; JETP Lett. 1996, 6, 471.

7. Kabala, Z. J.; Hunt, A.; Stochastic Hydrol. Hydraul. 1993, 7, 255.

8. Pilling, M. J.; Robertson, S. H.; Annu. Rev. Phys. Chem. 2003, 54, 245.

9. Robertson, S. H.; Pilling, M. J.; Jitariu, L. C.; Hillier, I. H.; Phys. Chem. Chem. Phys. 2007, 9, 4085.

10. Zhang, C.; Berry, R. S.; J. Chem. Phys. 2005, 123, 094103-1-10.

11. Tzenov, S. I.; Application of Master Equation Technique for the Study of Nonlinear Dynamics of Particles in Accelerators and Storage Rings, European Laboratory for Particles Physics, CERN Libraries: Geneva, 1992.

12. Kampen, N. V.; Stochastic Processes in Physics and Chemistry, $3^{\mathrm{a}}$ ed., North Holland: Amsterdam, 2007, cap. 5.

13. Salinas, S. R. A.; Introdução à Física Estatística, $2^{\mathrm{a}}$ ed., Ed. da USP São Paulo, 2005, cap. 16.3.

14. Anton, H.; Rorres, C.; Álgebra Linear com Aplicações, 8 a ed., Bookman: Porto Alegre, 2002, traduzido por Doering, I. C., cap. 11.6.
15. Zwanzig, R.; Nonequilibrium Statistical Mechanics, $1^{\mathrm{a}}$ ed., Oxford University Press: New York, 2001, cap. 3.

16. Jahnke, T.; Huisinga, W.; J. Math. Biol. 2007, 54, 1.

17. Tomé, T.; de Oliveira, M. J.; Dinâmica Estocástica e Irreversibilidade, $1^{\mathrm{a}}$ ed., Ed. da USP: São Paulo, 2001, cap. 6.

18. Nordsieck, A.; Lamb, W. E.; Uhlenbeck, G. E.; Physica 1940, 7, 344.

19. Oppenheim, I.; Shuler, K. E.; Weis, G. H.; Adv. Mol. Relax. Processes 1967, 1,13 .

20. Kemeny, J. G.; Snell, J. L. Em Finite Markov Chains: With a New Appendix “Generalization of a Fundamental Matrix" (Undergraduate Texts in Mathematics); Gehring, F. W.; Halmos, P. R., eds.; Springer-Verlag: New York, 1983, cap. 4.

21. Scherer, C.; Métodos Computacionais da Física, $1^{\mathrm{a}}$ ed., Ed. Livraria da Física: São Paulo, 2005, cap. 4.3.2.

22. Miller, J. A.; Klippenstein, S. J.; J. Phys. Chem. A 2003, 107, 7783.

23. Miller, J. A.; Klippenstein, S. J.; Georgievskii, Y.; Harding, L. B.; J. Phys. Chem. A 2010, 114, 4881.

24. Robinson, P. J.; Holbrook, K. A.; Unimolecular Reactions, $1^{\mathrm{a}}$ ed, Wiley: London, 1972.

25. Dunbar, R. C.; McMahon, T. B.; Thölmann, D.; Tonner, D. S.; Salahub, D. R.; Wei, D.; J. Am. Chem. Soc. 1995, 117, 12819.

26. Perrin, J.; Ann. Phys. 1919, 11, 5.

27. Dunbar, R. C.; McMahon, T. B.; Science 1998, 279, 194.

28. Eyler, J. R.; Mass Spectrom. Rev. 2009, 28, 448.

29. Dunbar, R. C.; J. Phys. Chem. 1994, 98, 8705.

30. Sena, M.; Riveros, J. M.; J. Phys. Chem. A 1997, 101, 4384. 